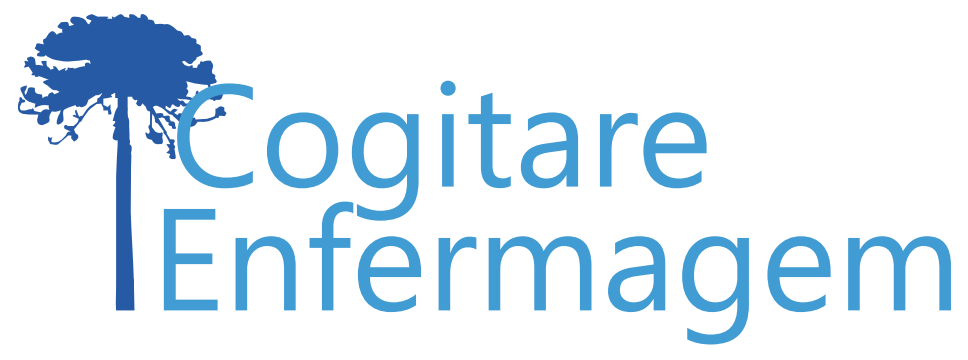

\title{
RECOMENDAÇÕES PARA O CUIDADO À CRIANÇA FRENTE AO NOVO CORONAVÍRUS
}

Sandra Teixeira de Araújo Pacheco ${ }^{1}$, Michelle Darezzo Rodrigues Nunes ${ }^{2}$, Juliana Zambrano Victória ${ }^{3}$, Welker da Silva Xavier ${ }^{4}$, Jaciane Alexandre da Silva $^{5}$, Cícero Ivan Alcantara Costa

\section{RESUMO}

Objetivo: identificar na literatura nacional e internacional as recomendações para o cuidado de crianças frente ao novo coronavírus.

Método: revisão integrativa, realizada no mês de abril de 2020, nas bases de dados Web of Science, CINAHL, BDENF, IBECS, LILACS, MEDLINE. Foram incluídos artigos de recomendação publicados em 2020. Excluíram-se estudos clínicos, sobre outras temáticas ou populações.

Resultados: a amostra foi constituída por 10 artigos, agrupados em três categorias de recomendações, que tratam: do cuidado a crianças com câncer; da busca pela diminuição dos malefícios biopsicossociais do confinamento; e das possibilidades de diagnóstico e tratamento.

Conclusão: as recomendações destacadas não se esgotam, mas representam um direcionamento para familiares/cuidadores e profissionais de saúde diante da grave situação. Para a enfermagem, conhecer as recomendações atuais sobre os cuidados a criança frente à COVID-19 possibilita a realização de ações para a promoção do cuidado de qualidade e seguro à criança.

DESCRITORES: Criança; Coronavírus; Covid-19; Enfermagem pediátrica; Enfermagem.

\section{COMO REFERENCIAR ESTE ARTIGO:}

Pacheco ST de, Nunes MDR, Victória JZ, Xavier W da S, Silva JA da, Costa CIA. Recomendações para o cuidado à criança frente ao novo corona vírus. Cogitare enferm. [Internet]. 2020 [acesso em "colocar data de acesso, dia, mês abreviado e ano"]; 25. Disponível em: http://dx.doi.org/10.5380/ce.v25i0.73554.

${ }^{1}$ Enfermeira. Doutora em Enfermagem. Docente de Graduação e Pós-Graduação em Enfermagem da Universidade do Estado do Rio de Janeiro. Rio de Janeiro, RJ, Brasil. $\odot$

${ }^{2}$ Enfermeira. Doutora em Ciências. Docente de Graduação e Pós-Graduação em Enfermagem da Universidade do Estado do Rio de Janeiro. Rio de Janeiro, RJ, Brasil. $\odot$

${ }^{3}$ Enfermeira. Mestranda em Enfermagem. Universidade do Estado do Rio de Janeiro. Rio de Janeiro, RJ, Brasil. $\odot$

${ }^{4}$ Enfermeiro. Residente em Enfermagem Pediátrica. Instituto Nacional de Saúde da Mulher, da Criança e do Adolescente. Rio de Janeiro, RJ, Brasil. $\odot$

${ }^{5}$ Enfermeira. Mestranda em Enfermagem. Técnica Universitária Superior no Hospital Universitário Pedro Ernesto. Rio de Janeiro, RJ, Brasil. $\odot$

${ }^{6}$ Enfermeiro. Doutorando em Enfermagem. Universidade do Estado do Rio de Janeiro. Rio de Janeiro, RJ, Brasil. $\odot$ 


\title{
RECOMMENDATIONS FOR CHILDCARE IN THE FACE OF THE NEW CORONAVIRUS
}

\begin{abstract}
Objective: To identify in the national and international literature the recommendations for the care of children against the new coronavirus.

Methods: An integrative review carried out in April 2020, in the Web of Science, CINAHL, BDENF, IBECS, LILACS, and MEDLINE databases. Recommendation articles published in 2020 were included. Clinical studies on other topics or populations were excluded.

Results: The sample consisted of 10 articles, grouped into three categories of recommendations, which deal with the following: the care for children with cancer; the search for the reduction of biopsychosocial harms in confinement; and the possibilities for diagnosis and treatment. Conclusion: The highlighted recommendations are not exhausted, but represent a guide for family members/caregivers and health professionals in the face of the severe situation. For nursing, knowing the current recommendations on child care in the face of COVID-19 makes it possible to carry out actions to promote quality and safe childcare.
\end{abstract}

DESCRIPTORS: Child; Coronavirus; Covid-19; Pediatric nursing; Nursing.

\section{RECOMENDACIONES PARA EL CUIDADO INFANTIL FRENTE AL NUEVO CORONAVIRUS}

\section{RESUMEN:}

Objetivo: identificar las recomendaciones para el cuidado infantil frente al nuevo coronavirus en la literatura nacional e internacional.

Métodos: revisión integradora realizada en el mes de abril de 2020, en las siguientes bases de datos: Web of Science, CINAHL, BDENF, IBECS, LILACS y MEDLINE. Se incluyeron artículos de recomendación publicados en 2020. Se excluyeron estudios clínicos sobre otros temas o poblaciones.

Resultados: la muestra estuvo compuesta por 10 artículos, agrupados en tres categorías de recomendaciones que abordan lo siguiente: el cuidado de niños con cáncer; la búsqueda por reducir los perjuicios psicosociales causados por el confinamiento; y las posibilidades de diagnóstico y tratamiento.

Conclusión: las recomendaciones destacadas no son exhaustivas pero sí representan una guía para familiares/cuidadores y profesionales de la salud frente a esta grave situación. Para la Enfermería, conocer las recomendaciones actuales sobre el cuidado infantil frente al COVID-19 permite llevar adelante acciones para promover un cuidado infantil seguro y de buena calidad.

DESCRIPTORES: Niño; Coronavirus; Covid-19; Enfermería pediátrica; Enfermería. 
No mundo, o início do ano de 2020 foi marcado com o aparecimento de uma doença causada por um novo coronavírus, o Severe Acute Respiratory Syndrome Coronavirus 2 (SARS-CoV-2), nome oficial atribuído pela Organização Mundial de Saúde ao Coronavírus da Síndrome Respiratória Aguda 2, causador da doença denominada COVID-19.

Até o momento, não há informações completas sobre a história natural da doença, restando ainda muitos pormenores a serem esclarecidos ${ }^{(1)}$. Porém, este vírus foi detectado em adultos pela primeira vez na China, na cidade de Wuhan em 31 de dezembro de 2019, e pela primeira vez em criança, no mesmo país, na cidade de Xangai em 19 de janeiro de $2020^{(2)}$.

Sabe-se que o vírus SARS-CoV-2 pertence à família coronaviridae. A maioria das infecções por este vírus é de baixa patogenicidade, entretanto pode eventualmente levar a infecções graves em pacientes imunodeprimidos, bem como afetar especialmente crianças, pacientes com comorbidades e idosos ${ }^{(3)}$.

Atualmente, as crianças são pouco afetadas pela doença em todo o mundo. A maioria delas apresenta a doença na forma assintomática ou leve. A maior magnitude relatada em crianças com doença grave foi de 10,6\% em menores de 1 ano, e 7,3\% em crianças entre 1 e 5 anos $^{(4)}$.

Apesar da população infantil ser menos atingida que a população adulta e apresentar na maioria das vezes a forma assintomática, elas são veículos de transmissão para os adultos, principalmente para os idosos, sendo o cuidado a essa clientela e a adoção de ações para impedir a propagação deste vírus importantes e necessários. Neste sentido, este artigo tem como objetivo descrever as recomendações publicadas na literatura nacional e internacional sobre os cuidados à criança frente à epidemia do novo coronavírus. Acredita-se que, através das publicações científica, os profissionais de saúde possam se instrumentalizar para planejar ações de cuidado e de educação em saúde para a criança e sua família, minimizando as consequências da doença e sua disseminação.

\section{MÉTODO}

Trata-se de uma revisão integrativa da literatura(5). Para sua realização, estabeleceramse os seguintes passos: identificação do tema e seleção da questão de pesquisa; estabelecimento de critérios para inclusão e exclusão de estudos; definição das informações a serem extraídas dos estudos selecionados e categorização dos estudos; avaliação dos estudos incluídos na revisão integrativa; interpretação dos resultados, apresentação da revisão; e síntese do conhecimento(b).

O tema escolhido foi recomendações acerca do COVID-19 para crianças e sua família, objetivando responder à seguinte questão: "Quais as recomendações publicadas acerca do cuidado à criança frente ao COVID-19?". Para a construção da pergunta adequada, utilizou-se a estratégia $\mathrm{PICO}^{(7)}$ - com " $\mathrm{P}$ " correspondendo à população (crianças de 2 a 12 anos); "I" à intervenção (recomendações); "C" à comparação (não se aplica, pois esse não é um estudo comparativo) e "O" correspondendo ao desffecho (cuidado).

A coleta de dados ocorreu em abril de 2020. As bases de dados pesquisadas foram MEDLINE® (via PubMed®), Web of Science e Cumulative Index of Nursing and Allied Health (CINAHL), Literatura Latino-Americana e do Caribe em Ciências da Saúde (LILACS), Índice Bibliográfico Español de Ciencias de la Salud (IBECS), e Banco de Dados em Enfermagem (BDENF). Para as buscas, utilizaram-se os descritores controlados "pré-escolar" (child, preschool), "criança" ("child") e "coronavírus" ("coronavirus"), identificados nos Descritores 
em Ciência da Saúde (DECs), Medical Subject Headings (MESH), e CINAHL Headings, além da palavra-chave: "COVID-19" ("2019-ncov"), com os operadores booleanos AND e OR. Em todas as bases de dados a estratégia utilizada para busca foi: (pré-escolar OR child) AND (coronavirus OR COVID-19) utilizados em inglês ou português a depender da base.

Foram incluídos artigos de recomendação, ou seja, artigos que trouxessem indicações ou advertências acerca do cuidado com crianças frente à COVID-19, cuja temática respondesse à pergunta norteadora, publicados em 2020, sem restrição de idioma. Excluíram-se estudos que focavam outras temáticas, realizados com população em faixa etária diferente da escolhida (neonatos, lactentes e adultos) e estudos clínicos.

Após as buscas, o primeiro passo foi a leitura dos títulos e dos resumos, entre dois autores de forma independente, para garantir que os textos atendiam à pergunta da revisão e aos critérios de inclusão. Como não houve discordância entre os dois autores, não foi necessário um terceiro leitor.

Para a extração e a síntese dos dados de cada estudo incluído na revisão, um quadro foi elaborado pelos autores, contendo as seguintes informações: título do artigo, país de origem, área de atuação dos autores, objetivos (se houvesse), participantes e principais recomendações e conclusões. Através dos dados incluídos no quadro, realizouse uma análise descritiva dos resultados, através de suas diferenças e as similaridades, sendo analisados criticamente e agrupados em três categorias de recomendações ${ }^{(8)}: 1$. Recomendações para crianças com câncer; 2 . Recomendações para a saúde biopsicossocial de crianças; 3 . Recomendações para o manejo do diagnóstico e tratamento da COVID-19.

\section{RESULTADOS}

Com as buscas nas bases de dados pesquisadas, 164 referências foram encontradas, sendo 124 na MEDLINE® (via PubMed $\otimes$ ), 16 na CINAHL, 24 na Web of Science e zero nas demais bases (LILACS, IBECS e BDENF). Após a leitura de títulos e resumos, foram excluídos 110 artigos. Dos 53 estudos selecionados para leitura na íntegra, 10 foram incluídos nos resultados desta revisão. A síntese da busca e os motivos de exclusão podem ser encontrados na Figura 1. 


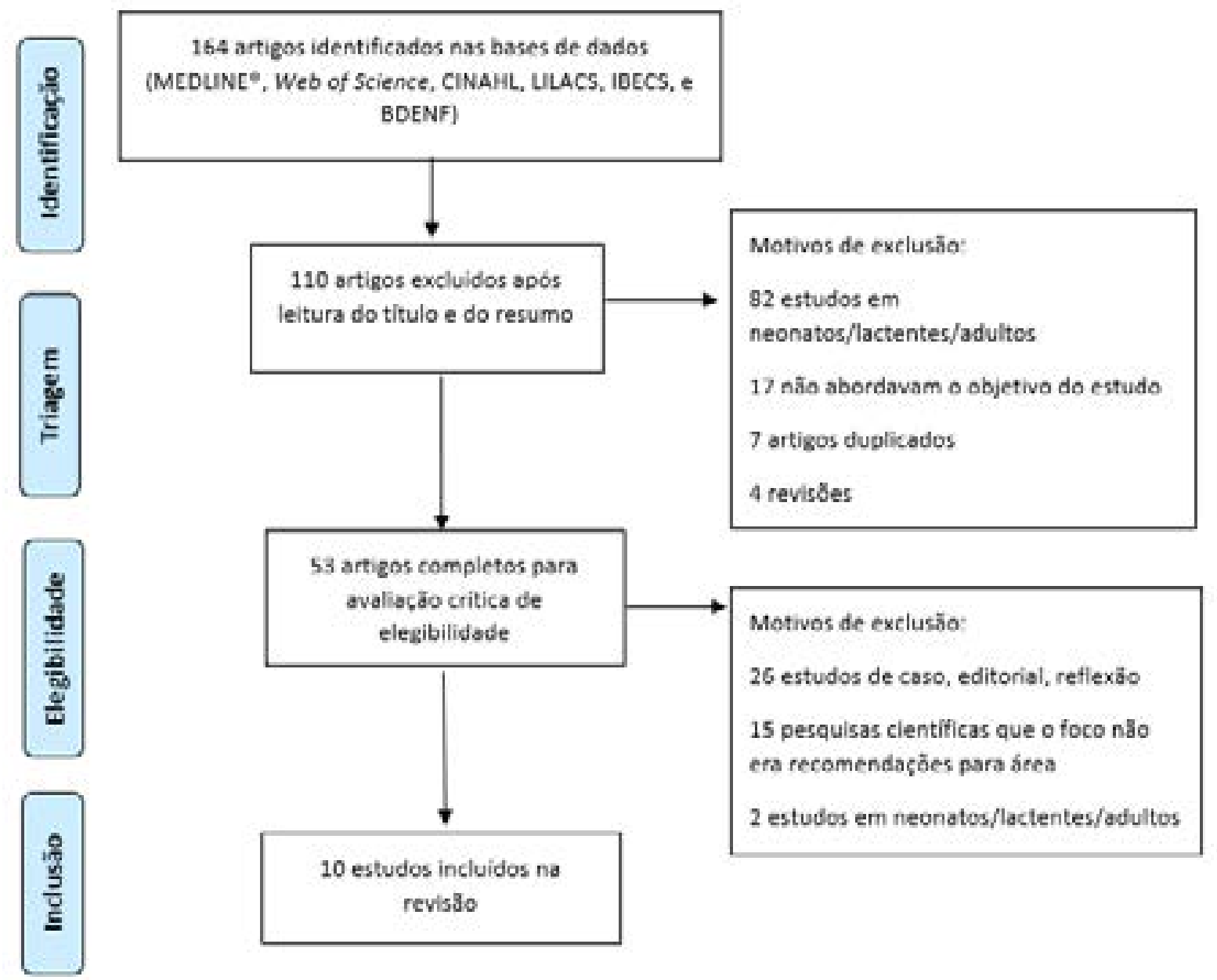

Figura 1 - Fluxograma segundo Preferred Reporting Items for Systematic Reviews and Meta-Analyses $(\text { PRISMA) })^{(9)}$. Rio de Janeiro, RJ, Brasil, 2020

Dentre os artigos selecionados, os países onde foram realizadas as pesquisas foram sete na China ${ }^{(10-16)}$, dois no Reino Unido ${ }^{(17,18)}$ e um na Itália(19).

Com relação à área de inserção dos autores, todos os estudos eram provenientes da área da medicina ${ }^{(10-19)}$, no entanto, dois deles eram especificamente da psiquiatria ${ }^{(17,19)}$ e um deles envolveu a parceria entre as áreas de medicina e enfermagem ${ }^{(18)}$.

Os estudos foram agrupados empiricamente, com base em seu conteúdo, sendo definidas 3 categorias de recomendações: Recomendações para crianças com câncer ${ }^{(10,13,18)}$; Recomendações para preservar a saúde mental de crianças e suas famílias ${ }^{(15-17,19) ;}$ e Recomendações para manejo clínico e diagnóstico(11,12,14).

\section{Recomendações para crianças com câncer}

Dos 10 estudos selecionados, três deles foram incluídos nesta categoria ${ }^{(10,13,18)}$, tendo como foco principal apresentar recomendações para crianças em tratamento de câncer durante o surto do novo coronavírus.

Os estudos ${ }^{(10,13,18)}$ destacam a necessidade de triagem nas estratégias: (1) Criar um sistema de informação para registro de casos, buscando aprimorar o conhecimento sobre o curso da doença em crianças com câncer e compartilhar experiências de gestão(10,13). (2) A criação de um site para a prestação de cuidados agendados, evitando a ida de crianças oncológicas ao ambiente hospitalar e não comprometendo os resultados do tratamento do 
câncer ${ }^{(18)}$. (3) Realizar antes da admissão uma triagem clínica para SARS-CoV-2, com base no critério presença de febre nas crianças oncológicas, considerando febre temperatura maior que $37,3 \circ \mathrm{C}$, durante três dias. Nos pacientes considerados suspeitos, encaminhar para a realização de exame tomográfico de pulmão e/ou teste de ácido nucléico ${ }^{(10)}$.

Nos casos altamente suspeitos ou confirmados de infecção por SARS-CoV-2, devese realizar a transferência para um hospital designado para infecção por SARS-CoV-2. Deve-se suspender imediatamente toda a quimioterapia da criança, e iniciar processos de trabalho relevantes e específicos da doença e implementar o isolamento. O tratamento quimioterápico só deve ser retomado após ações de investigação criteriosas(13).

Se, após a triagem, a criança oncológica apresentar os critérios negativos para SARSCoV-2, então deve-se iniciar o tratamento oncológico ${ }^{(10)}$. Nos casos de crianças com tumores hematológicos, deve-se realizar um diagnóstico diferencial de classificação de infecção por SARS-CoV-2, necessitando de investigações criteriosas: rotina sanguínea, proteína C reativa (PCR), ácido nucléico do vírus, indicadores bioquímicos e outros testes, se necessário. Assim, após excluir o COVID-19, elas podem ser admitidas para quimioterapia ${ }^{(13)}$.

Entretanto, nos tratamentos quimioterápicos e radioterápicos, entende-se que na quimioterapia é necessário a avaliação detalhada do estado clínico da criança e as considerações de riscos versus benefícios do procedimento, podendo acontecer uma redução moderada desse químico, ou o prolongamento do intervalo entre ciclos. Já o procedimento de radioterapia demonstra um menor risco comparado à quimioterapia, portando é razoável continuar com este tratamento(10).

Quanto às medidas de isolamento, todos os autores ${ }^{(10,13,18)}$ corroboram da opinião que crianças oncológicas devem permanecer em isolamento, tendo como justificativa a imunossupressão. Alguns autores salientam questões sobre a temática: (1) o isolamento deve ser administrado para os casos suspeitos e confirmados, e a criança só poderá receber o tratamento para o câncer após a remoção do isolamento(10). (2) Aplicar isolamento físico a todas as crianças com câncer em atual tratamento, tendo quarto individual em casa e durante a internação sempre que possível. Nos casos de internação, as visitas ambulatoriais devem ser limitadas e outros meios de comunicação devem ser priorizados, como ligações telefônicas e chamadas de vídeo ${ }^{(18)}$. Deve-se priorizar a transferência das crianças oncológicas internadas para o ambiente domiciliar ${ }^{(18)}$. (3) Quanto à circulação de pessoas: deve ser estabelecido limite de acesso nas enfermarias pediátricas oncológicas, tanto para profissionais circulantes quanto para pais e responsáveis, e, mesmo diante da diminuição do quantitativo, regras de distanciamento social devem ser estabelecidas, de pelo menos 1,8 metro entre indivíduos ${ }^{(18)}$. As visitas são proibidas e é permitida a permanência no setor somente de uma pessoa, a qual antes de entrar no hospital deve-se realizar investigação epidemiológica detalhada e verificar a temperatura corporal ${ }^{(13)}$.

O uso de equipamentos de proteção individual por familiares ainda é uma questão em debate ${ }^{(18)}$. Além disto, os estudos recomendam que as medidas de prevenção contra a infecção cruzada sejam intensificadas pelos profissionais de saúde, com implementação rigorosa de higienização das mãos, gerenciamento de resíduos e controle de infecção hospitalar ${ }^{(10,13,18)}$. Os autores ${ }^{(13)}$ trazem medidas mais rigorosas, como proibição de reunião de pessoas como jantares e demais eventos de confraternização; descanso de funcionários somente com o uso de máscara; e as roupas de uso das atividades laborais devem ser criteriosamente higienizadas diariamente.

Todos os funcionários dos setores pediátricos oncológicos devem medir a temperatura do corpo pelo menos uma vez por dia e seguir as recomendações de proteção pessoal: (1) Sala comum: máscaras cirúrgicas descartáveis, roupas de trabalho (revestimento branco), luvas de látex descartáveis. (2) Sala de isolamento para crianças oncológicas e realização de procedimento que possam gerar aerossóis em crianças suspeitas ou diagnosticadas: touca descartável, óculos de proteção ou máscara de proteção, máscara de proteção (N95), roupas de proteção descartáveis, luvas de látex descartáveis, sapatos de trabalho, capa impermeável para botas ${ }^{(13)}$. 
Por fim, os autores ${ }^{(10)}$ apontam que em situação de cirurgia, tanto em caso suspeito como confirmado, as fases pré-intra-pós devem ser realizadas de forma isolada, e a operação só deverá ser realizada em sala completamente desinfectada e com medidas de higiene e prevenção redobradas.

\section{Recomendações para a saúde biopsicossocial de crianças}

Dos 10 estudos selecionados, quatro deles foram incluídos nesta categoria(15-17,19), tendo como foco principal recomendações para mitigar os efeitos psicológicos causados nas crianças pelas orientações restritivas diante do COVID-19.

O mundo está focado em minimizar a transmissão do novo coronavírus, mas diante de diversas informações e mudanças abruptas na rotina, as crianças estão expostas a desenvolver altos níveis de estresse e ansiedade, devido às mudanças substanciais nos seus hábitos sociais e de lazer ${ }^{(17)}$. Diante desse panorama, é necessário orientar pais e cuidadores sobre ações sensíveis e eficazes que possam proteger seus filhos dessas angústias e ansiedades, proporcionando grandes benefícios no bem-estar psicológico infantil ${ }^{(17)}$. Além disso, crianças em confinamento são vulneráveis a riscos ambientais e de saúde física e mental, sendo necessário muita atenção e grandes esforços para atender a essas emergências efetivamente e evitar consequências em longo prazo ${ }^{(16)}$.

É apontado que, no confinamento, os pais são frequentemente os melhores e mais próximos recursos para as crianças procurarem ajuda. Assim, manter a comunicação com as crianças é a chave para identificar quaisquer questões físicas e psicológicas. Ressalta-se ainda que o confinamento pode oferecer uma boa oportunidade para melhorar a interação entre pais e filhos, envolvendo as crianças em atividades familiares, melhorando suas habilidades de autossuficiência e auxiliando em suas necessidades psicológicas ${ }^{(16)}$.

Os autores ${ }^{(16)}$ citam que as crianças estão constantemente expostas a notícias relacionadas à epidemia, então, ter conversas diretas sobre essas questões pode aliviar a ansiedade e evitar pânico ${ }^{(16)}$. Sendo assim, a companhia é essencial para o desenvolvimento psicológico e bem-estar das crianças, pois a separação de cuidadores proporciona a elas um estado de crise e pode aumentar o risco de distúrbios psiquiátricos ${ }^{(15)}$.

Outro ponto de destaque está na orientação adequada para as crianças. As crianças, mesmo pequenas (por exemplo, dois anos), percebem e sentem as mudanças ao seu redor, por estarem sintonizadas com os estados emocionais dos adultos e percebendo a ausência de possíveis cuidadores regulares, como avós e babás ${ }^{(17)}$. Portanto, é crucial a garantia de informações claras, precisas e honestas sobre as mudanças atuais, e se faz necessário que os adultos estejam vigilantes aos relatos das crianças, acolhendo e orientando-as ${ }^{(16)}$. Assim, pesquisadores sugerem aumentar o acesso das crianças às informações sobre a doença através de histórias em quadrinhos e vídeos, e orientar as crianças a estabelecer uma atividade regular ${ }^{(15)}$.

Para além disso, nas recomendações para preservação da saúde mental de crianças com a condição do espectro autista, o desafio pode ser ainda maior ${ }^{(19)}$. A condição do espectro autista é um distúrbio multifatorial grave com peculiaridades específicas nas áreas de comunicação social, interesses restritos e comportamentos repetitivos. Geralmente essas crianças fazem diversas intervenções semanais importantes para a manutenção do desenvolvimento cognitivo e motor, sendo neste momento impossibilitadas por causa da pandemia ${ }^{(19)}$.

Neste cenário de crianças com cuidados específicos, os autores indicam como estratégias de cuidado: a explicação clara do que é a COVID-19; formular uma estrutura das atividades da vida cotidiana; lidar com atividades lúdicas semiestruturadas, como uso de jogos e vídeo games; praticar terapias e consultas online, tanto com as crianças quanto com os responsáveis; e manter uma relação de contato mais estreito com a escola ${ }^{(19)}$.

Na rotina escolar, os autores apontam que é importante a escola garantir, segundo 
os requisitos educacionais, os conteúdos, e fornecer para as crianças diretrizes e princípios do aprendizado online. No entanto, também é importante não sobrecarregar os escolares, tendo em vista a sobrecarga natural da situação da doença ${ }^{(16)}$, fornecendo à criança uma estrutura das atividades diárias, proporcionando um equilíbrio no tempo de estudo e lazer $^{(19)}$. Sugere-se também que o governo possa mobilizar recursos existentes, criando uma plataforma para reunir os melhores cursos online sobre saúde, estilo de vida e apoio psicossocial disponíveis para as escolas ${ }^{(16)}$.

Como recomendações gerais, estudos apontam a prática de atividades físicas, dieta adequada, bons hábitos de sono, e se possível, procurar acompanhamento nutricional online para fornecer orientação e prevenir problemas cardíacos e de obesidade futuros devido ao sedentarismo ou pouca movimentação causados pelo confinamento ${ }^{(15,16)}$.

\section{Recomendações para o manejo do diagnóstico e tratamento da COVID-19}

Dos 10 estudos selecionados, três deles foram incluídos nesta categoria(11,12,14), cujo foco principal é fornecer recomendações referentes ao diagnóstico e o tratamento da COVID-19.

Com relação ao diagnóstico, recomenda-se realizar o diagnóstico diferencial precocemente, sendo o teste de ácido nucléico o principal método laboratorial utilizado e recomendado. Além desse, há: (1) o sequenciamento genético de amostras de swab, escarro, fezes ou sangue da garganta; e (2) a cultura dos grânulos de 2019-nCoV a partir de amostras de swab, escarro, fezes ou sangue na garganta ${ }^{(14)}$. Estes podem ser complementados com exames laboratoriais e de imagem, como rotina de sangue, indicadores de inflamação, bioquímica do sangue e fatores de coagulação, radiografia do tórax, tomografia de tórax, entre outros ${ }^{(12)}$.

Quanto ao tratamento, em geral, além de ser sintomático, por ainda não existir um medicamento antiviral eficaz para as crianças, baseia-se no isolamento e repouso. Cabe ressaltar a atenção para alterações nos sinais vitais e saturação de oxigênio para que, se necessário, sejam implementadas medidas efetivas de oxigenoterapia; a monitorização dos exames de sangue; o uso discriminado de antimicrobianos de amplo espectro; e a cautela no uso de corticosteroides de rotina ${ }^{(11,12,14)}$.

Para os casos graves, deve ser considerado suporte ventilatório e circulatório e devem ser internados em uma Unidade de Terapia Intensiva. Pode ser considerada a infusão de imunoglobulina intravenosa nos casos de resposta inflamatória sistêmica grave, e a terapia com Oxigenação por Membrana Extracorpória (ECMO) para preservar funções de órgãos, quando a ventilação mecânica, purificação do sangue e outras medidas são ineficazes ${ }^{(11,12,14)}$.

Para determinar o término do tratamento, a evolução do quadro em crianças é caracterizada por melhoria dos sintomas respiratórios, estabelecimento da temperatura corporal normal por três dias e dois testes de ácido nucleicos negativos, com intervalo de um dia entre eles ${ }^{(14)}$.

\section{DISCUSSÃO}

Atendendo ao objetivo do estudo, através desta revisão foi possível identificar as recomendações para o cuidado da criança frente à pandemia do novo coronavírus. As recomendações encontradas foram separadas em três categorias: a primeira referente ao cuidado as crianças com doenças onco-hematológicas, a segunda com relação à saúde mental e bem-estar da criança, e a última sobre as recomendações acerca do diagnóstico e tratamento da COVID-19.

Quanto à relevância para o cuidado à criança com câncer, um ponto importante 
a destacar é que as recomendações encontradas nesta revisão estiveram voltadas exclusivamente para as crianças em tratamento onco-hematológico, tendo como base sua imunossupressão e características terapêuticas especiais. Entretanto, existem diversas condições crônicas e necessidades especiais de saúde que tornam a criança vulnerável à infecção; além disso, os diversos tratamentos necessários são comprometidos ou interrompidos pelo isolamento social, o que também devem ser estudado e considerado em meio à pandemia.

Destaca-se que as crianças com condições crônicas ou necessidades especiais de saúde possuem demandas de cuidados contínuas, requerendo tratamento com frequentes visitas aos especialistas, ou hospitalizações para exames e tratamento ${ }^{(20,21)}$.

Outro ponto a ser discutido relaciona-se aos momentos em que a criança com COVID-19 precisa ficar hospitalizada. Segundo o Estatuto da Criança e do Adolescente, elas têm direito de ter um acompanhante durante todo o dia e noite ${ }^{(22)}$, porém, considerando a alta transmissibilidade do COVID-19, o uso de equipamentos de proteção individual por familiares/acompanhantes é uma questão importante e que ainda está em debate ${ }^{(18)}$.

Além disso, considerando as incertezas diante dos efeitos pessoais e globais neste cenário de pandemia, as recomendações voltadas para a preservação da saúde biopsicossocial da criança também foram foco de atenção dos estudos. Por esse motivo, pais e cuidadores são encorajados a estabelecer novas rotinas funcionais relacionadas à vida escolar, social e de lazer, porém dentro do ambiente domiciliar.

Ressalta-se que as novas exigências dos profissionais de saúde para o cuidar de um paciente predominantemente adulto com COVID-19 podem ampliar a invisibilidade para os casos de crianças e suas necessidades psicológicas urgentes ${ }^{(17)}$. Assim, autores sugerem que profissionais de saúde pediátrica recebam treinamento para facilitar a identificação precoce de problemas de saúde mental nas crianças, aprendendo a discernir comportamentos normais e anormais e verificando os sinais de alerta, como desconforto mental, preocupação, ansiedade, dificuldade no sono e perda de apetite. Também recomenda-se a padronização de informações na triagem da saúde mental, indicando a necessidade ou não de intervenção ou encaminhamento psiquiátrico e/ou terapêutico ${ }^{(15)}$.

Outro ponto que merece destaque é o fato de o Brasil ter adotado o isolamento social como uma das estratégias para conter a disseminação do vírus e, com isso, o fechamento total das escolas. Apesar de necessária, essa medida pode trazer riscos às crianças, como a interrupção do processo de aprendizagem e o aumento das taxas de abandono escolar, principalmente entre as famílias com algum tipo de vulnerabilidade social. Além disso, nesse contexto de vulnerabilidade, também pode ocorrer prejuízo na rede de apoio das crianças, afetando, por exemplo, o acesso a uma alimentação saudável e, a longo prazo, pode haver uma diminuição da parcela da população que é economicamente ativa ${ }^{(23)}$.

Dentre as estratégias para o enfrentamento desse problema, o ensino a distância tem ganhado grande força, mas a desigualdade social presente no mundo dificulta esse processo, visto que não são todos os países que possuem estruturas sólidas para promover uma boa educação, como dentro das diferentes regiões do Brasil. Logo, é importante se pensar em políticas educacionais para o período de pós-pandemia para diminuir os prejuízos daqueles alunos que não tiveram condições de manter o ensino a distância ${ }^{(23)}$.

Além disso, diante da necessidade de se permanecer em casa, é essencial um suporte familiar consistente para minimizar os prejuízos para o desenvolvimento das crianças, visto que tais situações de adversidade podem trazer consequências a curto prazo, como irritabilidade, distúrbios do sono e baixa imunidade, e também, a longo prazo, transtornos de ansiedade e depressão ${ }^{(24)}$.

Assim, a Sociedade Brasileira de Pediatria traz recomendações para esse momento de pandemia, tais como: os adultos devem manter momentos de diálogos e estabelecer uma rotina com as crianças; realizar orientações de higiene e cuidado com a saúde de maneira que a criança entenda, por meio de brincadeiras, por exemplo; conversar com os 
filhos sobre a real situação do mundo e não dizer que estão de férias, com uma linguagem clara e condizente com a idade; manter um estilo de vida saudável, com alimentaçã̃o e atividades físicas; estimular a criatividade e incentivar o ensino mesmo que a distância ${ }^{(24)}$.

Por fim, com relação às recomendações referentes ao diagnóstico e tratamento, destaca-se que a maioria das recomendações apresentadas na literatura segue as experiências vivenciadas com a população adulta, visto que ainda há uma limitação de casos e estudos pediátricos. Entretanto, com o aparecimento de novos casos e evidências, tais recomendações podem ser aprimoradas.

\section{CONCLUSÃO}

Os resultados desta revisão de literatura, divididos em três categorias, buscaram guiar as ações de familiares/cuidadores e dos profissionais de saúde no cuidado a crianças com câncer, diminuir os malefícios do confinamento em crianças e discutir possibilidades de diagnóstico e tratamento.

As recomendações destacadas não são exaustivas, mas representam um direcionamento para os familiares/cuidadores e profissionais de saúde diante da grave situação causada pela COVID-19. Destaca-se que, para a enfermagem, conhecer as recomendações atuais sobre os cuidados à criança frente ao novo coronavírus possibilita a realização de ações junto aos profissionais de enfermagem para garantir a promoção do cuidado de qualidade e seguro à criança.

Cabe ressaltar que se manter informado sobre as últimas atualizações e conhecer outras recomendações é fundamental para a saúde e proteção da criança e sua família, considerando o quão recente é a vivência dessa pandemia e o tamanho dos esforços que estão sendo desenvolvidos para guiar nossa forma de lidar com o novo coronavírus em crianças.

\section{REFERÊNCIAS}

1. Ministério da Saúde (BR). Secretaria de Atenção Primária à Saúde (SAPS). Protocolo Clínico do Coronavírus - COVID-19 na atenção primária em saúde. [Internet]. 2020 [acesso em 20 abr 2020]; Disponível em: https://www.saude.gov.br/images/pdf/2020/marco/20/20200318-ProtocoloManejover002.pdf.

2. Cai JH, Wang XS, Ge YL, Xia AM, Chang HL, Tian H, et al. First case of 2019 novel coronavirus infection in children in Shanghai. Pub Med [Internet]. 2020 [acesso em 25 abr 2020]; 58(2). Disponível em: https:// pubmed.ncbi.nlm.nih.gov/32023679/.

3. Ministério da Saúde (BR). Secretaria de Vigilância em Saúde. Doença pelo Novo Coronavírus 2019 COVID-19. Boletim epidemiológico 03. [Internet]. 2020 [acesso em 20 abr 2020]; Disponível em: https:// portalarquivos2.saude.gov.br/images/pdf/2020/fevereiro/21/2020-02-21-Boletim-Epidemiologico03.pdf.

4. Sociedade Brasileira de Peneumologia e Tisiologia. COVID-19: orientações da SBPT sobre o tratamento de crianças [Internet]. 2020 [acesso em 20 abr 2020]. Disponível em: https://sbpt.org.br/ portal/wp-content/uploads/2020/04/pneumopediatria covid 19.pdf.

5. Whittemore R, Knafl K. The integrative review: updated methodology. J Adv Nurs. [Internet]. 2005 [acesso em 20 abr 2020]; 52(5). Disponível em: https://www.ncbi.nlm.nih.gov/pubmed/16268861.

6. Mendes KDS, Silveira RC de CP, Galvão CM. Revisão integrativa: método de pesquisa para a incorporação de evidências na saúde e na enfermagem. Texto contexto - enferm. [Internet]. 2008 [acesso 
em 20 abr 2020]; 17(4). Disponível em: http://dx.doi.org/10.1590/S0104-07072008000400018.

7. Santos CM da C, Pimenta CA de M, Nobre MRC. The PICO strategy for the research question construction and evidence search. Rev. Latino-Am. Enfermem [Internet]. 2007 [acesso em 20 abr 2020]; 15(3). Disponível em: http://dx.doi.org/10.1590/S0104-11692007000300023.

8. Cooper HM. Scientific Guidelines for Conducting Integrative Research Reviews. Review of Educational Research. [Internet]. 1982 [acesso em 20 abr 2020]; 52. Disponível em: https://doi. org/10.3102\%2F00346543052002291.

9. Moher D, Liberati A, Tetzlaff J, Altman DG. Preferred reporting items for systematic reviews and metaanalyses: the PRISMA statement. PLoS Med. [Internet]. 2009 [acesso em 20 abr 2020]; 6(7). Disponível em: https://doi.org/10.1371/journal.pmed.1000097.

10. Yang C, Li C, Wang S. Clinical strategies for treating pediatric cancer during the outbreak of 2019 novel coronavirus infection. Pediatr Blood Cancer. [Internet]. 2020 [acesso em 25 abr 2020];67(5). Disponível em: https://doi.org/10.1002/pbc.28248.

11. Pediatric Branch of Hubei Medical Association, Pediatric Branch of Wuhan Medical Association, Pediatric Medical Quality Control Center of Hubei. Recommendation for the diagnosis and treatment of novel coronavirus infection in children in Hubei (Trial version 1). Zhonghua Er Ke Za Zhi. [Internet]. 2020 [acesso em 25 abr 2020]; 22(2). Disponível em: https://doi.org/10.7499/j.issn.1008-8830.2020.02.003.

12. Society of Pediatrics, Chinese Medical Association, Editorial Board, Chinese Journal of Pediatrics. Recommendations for the diagnosis, prevention and control of the 2019 novel coronavirus infection in children (first interim edition). Zhonghua Er Ke Za Zhi. [Internet]. 2020 [acesso em 25 abr 2020]; 58(0). Disponível em: https://doi.org/10.3760/cma.j.issn.0578-1310.2020.0004.

13. Subspecialty Group of Hematology and Oncology, Society of Pediatrics of Hubei. Standardized management guideline for pediatric wards of hematology and oncology during the epidemic of coronavirus disease 2019. Zhongguo Dang Dai Er Ke Za Zhi. [Internet]. 2020 [acesso em 25 abr 2020]; 22(3). Disponível em: https://pubmed.ncbi.nlm.nih.gov/32204750/.

14. Chen ZM, Fu JF, Shu $\mathrm{Q}$, Chen YH, Hua CZ, Li FB, et al. Diagnosis and treatment recommendations for pediatric respiratory infection caused by the 2019 novel coronavirus. World J Pediatr [Internet]. 2020 [acesso em 21 abr 2020]; Disponível em: https://doi.org/10.1007/s12519-020-00345-5.

15. Liu JJ, Bao Y, Huang X, Shi J, Lu L. Mental health considerations for children quarantined because of COVID-19. Lancet Child Adolesc Health. [Internet]. 2020 [acesso em 25 abr 2020]; 4(5). Disponível em: https://doi.org/10.1016/S2352-4642(20)30096-1.

16. Wang G, Zhang Y, Zhao J, Zhang J, Jiang F. Mitigate the effects of home confinement on children during the COVID-19 outbreak. Lancet. [Internet]. 2020 [acesso em 25 abr 2020]; 395(10228). Disponível em: https://doi.org/10.1016/S0140-6736(20)30547-X.

17. Dalton L, Rapa E, Stein A. Protecting the psychological health of children through effective communication about COVID-19. The Lancet Child Adolesc Health [Internet]. 2020 [acesso em $21 \mathrm{abr}$ 2020]; 4(5). Disponível em: https://www.thelancet.com/journals/lanchi/article/PIIS2352-4642(20)30097-3/ abstract.

18. Bouffet E, Challinor J, Sullivan M, Biondi A, Rodriguez-Galindo C, Pritchard-Jones K. Early advice on managing children with cancer during the COVID-19 pandemic and a call for sharing experiences. Pediatr Blood Cancer. [Internet]. 2020 [acesso em 25 abr 2020]; 67(7). Disponível em: https://doi.org/10.1002/ pbc. 28327.

19. Narzisi A. Handle the autism spectrum condition during Coronavirus (COVID-19) stay at home period: ten tips for helping parents and caregivers of young children. Brain Sci. [Internet]. 2020 [acesso em $25 \mathrm{abr}$ 2020]; 10(4). Disponível em: https://doi.org/10.3390/brainsci10040207.

20. Hedén L, Pöder U, Essen L, Ljungman G. Parents' perceptions of their child's symptom burden during and after cancer treatment. J. Pain Symptom Manage. [Internet]. 2013 [acesso em 10 maio 2020]; 46(3). 
Disponível em: http://dx.doi.org/10.1016/j.jpainsymman.2012.09.012.

21. Silveira A da, Neves ET. Vulnerabilidade das crianças com necessidades especiais de saúde: implicações para a enfermagem. Rev Gaúcha Enferm. [Internet]. 2012 [acesso em 09 maio 2020]; 33(4). Disponível em: https://doi.org/10.1590/S1983-14472012000400022.

22. Brasil. Lei n. 8.069, de 13 de julho de 1990, e legislação correlata. Estatuto da Criança e do Adolescente. 9.ed. [Internet]. Brasília: Série Legislação; 2012 [acesso em 30 abr 2020]; Disponível em: http://www.crianca.mppr.mp.br/arquivos/File/publi/camara/estatuto crianca adolescente 9ed.pdf.

23. Banco Mundial. Políticas Educacionais na Pandemia da COVID-19: o que o Brasil pode aprender com o resto do mundo? [Internet]. World Bank Group. [acesso em 20 maio 2020]. Disponível em: https://www. worldbank.org/pt/country/brazil/publication/brazil-education-policy-covid-19-coronavirus-pandemic.

24. Sociedade Brasileira de Pediatria (SBP). Departamento Científico de Pediatria do Desenvolvimento e Comportamento. Pais e filhos em confinamento durante a pandemia de COVID-19 [Internet]. 2020 [acesso em 20 maio 2020]. Disponível em: https://www.sbp.com.br/imprensa/detalhe/nid/pais-e-filhosem-confinamento-durante-a-pandemia-de-covid-19/.

Recebido: 07/05/2020

Finalizado: 10/06/2020

Editora associada: Luciana Puchalski Kalinke

Autor Correspondente:

Michelle Darezzo Rodrigues Nunes

Universidade do Estado do Rio de Janeiro

Bv. 28 de Setembro, 157 - 20551-030 - Rio de Janeiro, RJ, Brasil

E-mail: mid13@hotmail.com

Contribuição dos autores:

Contribuições substanciais para a concepção ou desenho do estudo; ou a aquisição, análise ou interpretação de dados do estudo - JZV, WSX, JAS, CIAC

Aprovação da versão final do estudo a ser publicado - STAP, MDRN, JZV, WSX, JAS, CIAC

Responsável por todos os aspectos do estudo, assegurando as questões de precisão ou integridade de qualquer parte do estudo - STAP, MDRN

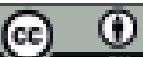

Este obra está licenciado com uma Licença Creative Commons Atribuição 4.0 Internacional. 